\title{
LV. On some effects of dissociation on the physical properties of gases
}

\author{
W.M. Hicks M.A.
}

To cite this article: W.M. Hicks M.A. (1877) LV. On some effects of dissociation on the physical properties of gases, Philosophical Magazine Series 5, 3:20, 401-418, DOI: 10.1080/14786447708639262

To link to this article: http://dx.doi.org/10.1080/14786447708639262

曲 Published online: 13 May 2009.

Submit your article to this journal $[\pi$

Џ Article views: 2

Q View related articles $\asymp$ 
LONDON, EDINBURGH, AND DUBLIN

\section{PHILOSOPHICAL MAGAZINE}

AND

JOURNAL OF SCIENCE.

[FIFTH SERIES.]

$J U N E 1877$.

LV. On some Effects of Dissociation on the Physical Properties of Gases. By W. M. Hrcks, M.A., Fellow of St. John's College, Cambridge*.

THE following pages contain an attempt to discover what 1 effect the dissociation of an elementary or compound gas has upon its physical constants. That dissociation must exist to some extent at all temperatures is exceedingly probable; and if so, it must necessarily affect the physical properties of the gas; and especially we might suppose that it would produce variation from Charles's law, and perhaps explain the difficulty hitherto experienced in accounting for the ratio $1 \cdot 408$ of the specific heats of a permanent gas. It was under this belief, and also with some hope of throwing a little light on the chemical changes which take place in compound gases, and on mixing different gases, that I undertook the mathematical investigation of it.

I.

1. Before we can apply mathematical reasoning to the consideration of dissociation, it will be necessary to have some hypothesis on the manner in which it takes place. Our hypothesis should be one which, while being as simple as possible, is likely to contain the essentials of what really takes place, even though it may not be correct in all its details. Dissociation of a compound gas is that state in which the compound molecules of a gas are split up into their component parts and exist together uncombined. I shall suppose the same also to

* Communicated by the Author.

Phil. Mag. S. 5. Vol. 3. No. 20. June 1877. 
take place in an elementary gas, the molecule being composed of two atoms. How the atoms are bound together we do not know ; but, from what we can gather, there seems to be some attractive force between them which at very close quarters changes into a repulsive one. Equilibrium is sustained by the attraction between the two atoms and their motion about one another. If, then, the two atoms of a molecule become separated, there seem only two ways of accounting for it. Either their relative motion becomes so large as to overcome the force of attraction; or some external force must act upon them, which can be nothing else than a reaction between them and some other molecule. The latter is the hypothesis I have adopted in the following investigation.

2. I consider the atom to be smooth, spherical, and perfectly elastic, and, in order to bring the dissociation under mathematical treatment, suppose

(1) That when a molecule experiences a blow greater than a certain blow $c$, it breaks up into its component atoms,

(2) That when two atoms impinge with a blow less than $c$, they combine to form a molecule.

Now it is exceedingly improbable that any of these suppositions is absolutely true ; but yet I venture to think, since the mathematical form would be similar, that the state of such a gas would differ only slightly from that of real gases. As was said before, the reaction between two molecules is probably a varying one, and it is unlikely that they ever come into real contact; still the mean effect will be similar to the case under our hypothesis. We nust look upon $c$ as a mean blow for different directions of incidence, or as some quantity which in the real state determines whether the molecules will break up or not. For instance, if the force between two atoms were inversely as the square of the distance, $c$ would determine whether the resulting orbit of two atoms coming together would be an ellipse, or a parabola or hyperbola. So also the radii of action must be taken as average quantities. Further, it is not likely that they are absolutely independent of the temperature; for it is conceivable that as the internal energy increases they will fly further apart; and thus become more liable to blows from the other molecules. Neither are we perhaps warranted in assuming that $c$ is constant; since $c$ constant involves the invariability of the distance of the two atoms; for if the distance increased, the force between would diminish, and therefore $c$ also. Nevertheless, although numerical results would be affected by this cause, the general laws would be of the same form in the two cases; and in either case our experimental knowledge is neither wide 
enough, nor exact enough, to enable us to deduce exact numerical results.

3. In the present paper I first consider the problem, To find the number of blows greator than $c$, between two sets of moving particles, per unit of time, in unit of volume. This is then applied to find the dissociation at any temperature, and a quadratic obtained, the positive root of which gives the ratio of molecules to free atoms in the gas ; and it is shown that the resulting state of the gas is a stable one. This ratio is used to investigate the relation between the temperature and pressure; and it is shown that Charles's law is not rigorously exact, though within ordinary ranges of temperature it is very nearly so ; reasons are also given why for the permanent gases $c$ should be large, compared with the mean blow at ordinary temperatures. Next the specific heats are considered; and it is proved that for a diatomic molecule, in which the atoms are smooth and spherical, or the energy of rotation of the atoms is unaffected by external causes, the ratio of the specific heats is about 1.4 ; it is also shown that the specific heats are almost rigorously constant at ordinary temperatures. This concludes the present paper ; in what is to follow I intend to discuss the properties of a compound gas of the type $\mathrm{HCl}$.

4. When we pass to the consideration of a compound gas, even of the simplest form, additional difficulties are introduced, as equations of a high order appear whose algebraical solution is impossible, and from which therefore it will be extremely difficult to deduce general laws. All we seem able to do is to take one or two particular cases, and learn what we can about them. For instance, in the case of a gas of the type $\mathrm{HCl}$, i.e. in which two monatomic gases combine to form a gas whose molecule is diatomic, we get three equations of the second degree between three unknowns. In general, when two gases $A$ and $B$ are nixed together, there will always be a certain proportion of a new gas $\mathrm{C}$ whose molecule is composed of atoms of $A$ and $B$, and the proportions of free atoms and molecules of $A$ and $B$ will be altered. When the excess of the number of combinations of free atoms of A with $B$ over the number of molecules of $\mathrm{C}$ destroyed in any time is greater than the number of combinations of free atoms of $A$ to form $A$ and of free atoms of $B$ to form $B$, then the proportion of $A$ and $B$ decreases, whilst that of $C$ increases, and we get a chemical change. This will explain why often the mere presence of another gas $\mathrm{D}$ will produce a change of $\mathrm{A}$ and $\mathrm{B}$ into $\mathrm{C}$; for the action of $\mathrm{D}$ on $\mathrm{A}$ and $\mathrm{B}$ may cause more of their molecules to be broken up than of $\mathrm{C}$, while at the same $2 \mathrm{D} 2$ 
time not many more of its own are ; therefore, since the combinations of $\mathrm{A}$ and $\mathrm{B}$ atoms into $\mathrm{C}$ molecules are destroyed more slowly than the combinations of $\mathrm{A}$ and $\mathrm{B}$ atoms into $\mathrm{A}$ and $\mathrm{B}$ molecules, the gases $\mathrm{A}$ and $\mathrm{B}$ after a time change into $\mathrm{C}$.

5. In a similar manner we may explain the result obtained by Andrews, that if nitrogen be mixed with carbon dioxide, the mixture may be subjected to very high pressure without the carbon dioxide being condensed. When a gas condenses, we must suppose that molecules combine with molecules to form larger aggregations, and that this continues until the whole gas is condensed into a liquid. When the temperature is below a certain degree, the change from the gaseous to the liquid state takes place around nuclei, in which the molecules get entangled, and which the energy of agitation is not great enough to destroy; in this case a mist is formed, and we see the gas condense. But when the temperature is higher than a certain degree (the critical temperature), the energy of agitation is so great that an aggregation greater than the average gets broken up as soon as formed, and the gas passes into the liquid form by having its particles uniformly pressed more closely together; no separate nuclei are formed to diffuse the light passing through, and we can see no change take place. The nitrogen molecules and atoms act in the same way to break up the carbon dioxide aggregations as soon as formed, and hence prevent it from condensing.

6. The fact that two states of a gas are possible under the same conditions, as for instance $2 \mathrm{~N}+\mathrm{O}$ and $\mathrm{N}_{2} \mathrm{O}$, is easily explicable, as it is quite clear that in general there will bo more than one way in which the losses of the different molecules may be equal to their gains. In other words, the equations of high order, which we obtain, have several positive roots, which give a stable state of the gas: as the temperature rises, one such state may become unstable; in this case we shall get a sudden change into one of the other stable states, with a transformation of energy, answering in experience to an explosion with evolution of heat.

\section{II.}

7. Let $\mathrm{N}$ be the number of molecules of mass $m_{1}$ in unit of volume, and $\mathbf{N}^{\prime}$ the number of molecules of mass $m_{2}$. Also let $\alpha, \beta$ be the velocities of mean square in the two systems respectively, and $s$. the mean effective distance between molecules of the first kind and of the second.

Consider a molecule of the first kind moving with velocity $v$; the number of such whose velocities lie between $v$ and $v+\delta v$ 
is $\frac{4 \mathrm{~N}}{\sqrt{\pi} \alpha^{3}} v^{2} e^{-\frac{v^{2}}{\alpha^{2}} \delta v^{*}}$; and let us find the number of collisions in unit of time batween one of thess $\left(m_{1}\right)$ and the second system of molecules. The number of molecules of the second kind in unit of volume whose velocities lie between $u$ and $u+\delta u$ is $\frac{4 \mathrm{~N}^{\prime}}{\sqrt{\pi \beta^{3}}} u^{2} e^{-\frac{u^{2}}{\beta^{2}}} \delta u$. The proportion of those moving at angles between $\theta$ and $\theta+\delta \theta$ with the direction of motion of $m_{1}$ is $\frac{1}{2} \sin \theta \delta \theta$.

The relative velocity of $m_{1}$ with these

$$
=\sqrt{u^{2}+v^{2}+2 u v \cos \bar{\theta}}=r, \text { say ; }
$$

and the number of collisions in unit of time is

$$
\pi s^{2} r \cdot \frac{4 \mathrm{~N}^{\prime}}{\sqrt{\pi} \beta^{3}} u^{2} e^{-\frac{u^{2}}{\beta^{2}}} \cdot \frac{1}{2} \sin \theta \delta u \delta \theta=\overline{\mathrm{N}} r \sin \theta d \theta, \text { say. }
$$

8. We have now to find the proportion of those that strike with a blow greater than a given quantity $(c$, say). Consider a molecule of the second system striking $m_{1}$ at an arcual distance $\phi$ from the point of direct impact. The relative velocity of the surfaces before impact is $r \cos \phi$, and as the molecules are supposed perfectly elastic, the blow will be $\frac{2 m_{1} m_{2}}{m_{1}+m_{2}} r \cos \phi$; and this must be $>c$. If, therefore, $\phi_{1}$ be such an angle that $2 m_{1} m_{2} r \cos \phi_{1}=\left(m_{1}+m_{2}\right) c$, all those molecules that fall within a small circle whose radius is $s \sin \phi_{1}$ will strike with a blow $>c$. The proportion required of the whole number of impacts is therefore

$$
\frac{\pi s^{2} \sin ^{2} \phi_{1}}{\pi s^{2}}=\sin ^{2} \phi_{1}=1-\left(\frac{\left.m_{1}+m_{2}\right) c}{2 m_{1} m_{2} r}\right)^{2}=1-\left(\frac{c^{\prime}}{r}\right)^{2}, \text { say ; }
$$

and the whole number of collisions required

$$
=\overline{\mathrm{N}} r\left\{1-\left(\frac{c^{r}}{r}\right)^{2}\right\} \sin \theta \delta \theta .
$$

9. The number of molecules of the second kind with velocities between $u$ and $u+\delta u$, and making an angle between $\theta$ and $\theta+\delta \theta$ with direction of $m_{1}$, is

$$
\frac{2 \mathrm{~N}^{\prime}}{\sqrt{ } \bar{\pi} \beta^{3}} \sin \theta \delta \theta u^{2} e^{-\frac{u^{2}}{\bar{\beta}^{2}} \delta u} \text {. }
$$

Therefore the number of those with velocity $u$ which have a

* See Maxwell, Phil. Mag. January 1860. 
406 Mr. W. M. Hicks on some Effects of Dissaciation

velocity $r$ relative to $m_{1}$

where

$$
=\frac{2 N^{\prime}}{\sqrt{\pi} \beta^{3}} \sin \theta_{1} \delta \theta u^{2} e^{-\frac{u^{2}}{\beta^{2}}} \delta u,
$$

$$
u^{2}+v^{2}+2 u v \cos \theta_{1}=r^{2} .
$$

For the same value of $u$ when $r$ becomes $r+\delta r, \theta_{1}$ becomes $\theta_{1}+\delta \theta_{1}$, where

$$
\sin \theta_{1} \delta \theta_{1}=-\frac{r}{u v} \delta r
$$

and the above number becomes

$$
-\frac{2 N^{\prime} u}{\sqrt{ } \pi \beta^{3} v} e^{-\frac{u^{2}}{\beta^{2}} \delta u r \delta r .}
$$

To find the number of molecules impinging on $m_{1}$ with relative velocity between $r$ and $r+\delta r$, this must be multiplied by $\pi s^{2} r$ and integrated with respect to $u$ over all possible values of $u$, i. e. for such that $\frac{r^{2}-u^{2}-v^{2}}{2 u v}$ lies between \pm 1 , or

$$
\begin{aligned}
& u+v>r, \\
& u \sim v<r .
\end{aligned}
$$

The number required is therefore

$$
\begin{aligned}
& =\frac{2 \sqrt{\pi} N^{\prime} s^{2} r^{2}}{\beta^{3} v} \delta r \int_{r-v}^{r+v} u e^{-\frac{u^{2}}{\beta^{2}}} d u \\
& =\frac{\sqrt{\pi} N^{\prime} s^{2} r^{2}}{\beta v} \delta v\left\{e^{-\frac{(r-v)^{2}}{\beta^{2}}}-e^{-\frac{(r+v))^{2}}{\beta z}}\right\} d v .
\end{aligned}
$$

The proportion of those impinging with a blow $>c$ is $1-\frac{e^{\prime 2}}{r^{2}}$. Hence the whole number of impacts of the second system on $m_{1}$ with a blow $>c$ is

$$
\frac{\sqrt{\pi} \mathrm{N}^{\prime} s^{2}}{\beta v} \int_{\sigma^{\prime}}^{\infty}\left(r^{2}-c^{\prime 2}\right)\left\{e^{-\frac{(r-v)^{2}}{\beta^{2}}}-e^{-\frac{(r+v)^{2}}{\beta^{2}}}\right\} d r ;
$$

and the whole number of blows $>e$ beiween molecules of the first and second kinds per unit of time is

$$
\frac{4 \mathrm{NN}^{\prime} s^{2}}{\alpha^{3} \beta} \int_{0}^{\infty} \int_{c^{\prime}}^{\infty}\left(r^{2}-c^{\prime 2}\right)\left\{e^{-\frac{(r-v)^{2}}{\beta^{2}}}-e^{-\frac{(r+v)^{2}}{\beta}}\right\} v e^{-\frac{v^{2}}{\alpha^{2}}} d v d r .
$$

This may be put in the form

$$
\frac{4 \mathrm{NN} N^{\prime} s^{2}}{a^{3} \beta} \int_{c^{\prime}}^{\infty}\left(r^{2}-c^{\prime 2}\right) e^{-\frac{r^{2}}{a^{2}+\beta^{2}}} d r \int_{0}^{\infty}\left\{e^{-\frac{(v-h) 2}{\gamma^{2}}}-e^{-\frac{(v+h) 2}{\gamma^{2}}}\right\} v d v
$$


where

$$
h=\frac{\alpha^{2} r}{\alpha^{2}+\beta^{2}} \text { and } \gamma^{2}=\frac{\alpha^{2} \beta^{2}}{\alpha^{2}+\beta^{2}} .
$$

The second integral

$$
=\int_{-\infty}^{+\infty}(v+h) e^{-\frac{v^{2}}{\gamma^{2}}} d v=h \sqrt{\pi} \gamma=\frac{\sqrt{\pi} \alpha^{3} \beta r^{v}}{\left(\alpha^{2}+\beta^{2}\right)^{\frac{3}{2}}}
$$

Hence the whole integral

$$
\begin{aligned}
& =\frac{4 \sqrt{\pi} N N^{\prime} s^{2}}{\left(\alpha^{2}+\beta^{2}\right)^{\frac{3}{2}}} \int_{c^{\prime}}^{\infty}\left(r^{2}-c^{\prime 2}\right) r e^{-\frac{r^{2}}{\alpha^{2}+\beta^{2}}} d r \\
& =2 \sqrt{\pi} N N^{\prime} s^{2} \sqrt{\alpha^{2}+\beta^{2}} e^{-\frac{c^{\prime 2}}{\alpha^{2}+\beta^{2}}} .
\end{aligned}
$$

Let $\frac{\theta}{2}$ be the common mean kinetic energy of molecules in each system. Then

also

$$
\begin{gathered}
m_{1} \alpha^{2}=m_{2} \beta^{2}=\theta ; \\
\therefore \alpha^{2}+\beta^{2}=\frac{m_{1}+m_{2}}{m_{1} m_{2}} \theta ;
\end{gathered}
$$

$$
c^{\prime}=\frac{m_{1}+m_{2}}{2 m_{1} m_{2}} c .
$$

Hence the number of blows $>c$ in a unit of volume per unit of time between molecules of the first and second kinds respectively is

$$
2 \sqrt{\pi} \mathrm{NN}^{\prime} s^{2} \sqrt{\frac{m_{1}+m_{2}}{m_{1} m_{2}}} \sqrt{ } \bar{\theta} e^{-\frac{m_{1}+m_{2}}{4 m_{1} m_{2}} \cdot \frac{c^{2}}{\theta}}
$$

The law of variation of the number for the same pair of gases is of the form

$$
\Delta \rho \rho^{\prime} \sqrt{t} e^{-\frac{t_{0}}{t}},
$$

where $\rho, \rho^{\prime}$ are the densities of the two gases, $t$ is the absolute temperature, and $\mathrm{A}$ and $t_{0}$ are constants. It is probable that $c$ or $t_{0}$ will vary with the amount of internal energy of the molecules, and will diminish as the temperature increases.

Putting $c=0$, we find the whole number of collisions to be

$$
2 \sqrt{\pi} \mathrm{NN}^{\prime} s^{2} \sqrt{\frac{m_{1}+m_{2}}{m_{1} m_{2}}} \sqrt{\theta}
$$

as has been shown by Maxwell*. Hence the number of colli-

* Phil. Mag. January 1860 
sions which impinge with a blow $<0$

$$
=2 \sqrt{\pi} \mathrm{NN}^{\prime} s^{2} \sqrt{\frac{m_{1}+m_{2}}{m_{1} m_{2}}}\left(1-e^{-\frac{m_{1}+m_{2}}{4 m_{1} m_{2}} \cdot \frac{c^{2}}{\theta}}\right) \sqrt{\theta} .
$$

10. The foregoing expression enables us to find the mean blow at any temperature, thus. The number of blows $>c$ is given by an expression of the form $\lambda e^{-\mu c^{2}}$; hence the number of blows between $c$ and $c+\delta c$

$$
=2 \lambda \mu c e^{-\mu c^{2}} \delta c ;
$$

hence the mean blow

$$
\begin{aligned}
= & \frac{2 \lambda \mu \int_{0}^{\infty} c^{2} e^{-\mu c^{2}} d c}{\text { No. of blows }>0} \\
= & \frac{2}{\sqrt{\mu}} \int_{0}^{\infty} x^{2} e^{-x^{2}} d x=\frac{1}{\sqrt{\mu}} \int_{0}^{\infty} e^{-x^{2}} d x=\frac{1}{2} \sqrt{\frac{\pi}{\mu}} ;
\end{aligned}
$$

hence, calling the mean blow $c^{\prime}$,

$$
c^{\prime 2}=\frac{\pi}{4 \mu}=\frac{\pi}{4} \cdot \frac{4 m_{1} m_{2}}{m_{1}+m_{2}} \theta=\pi \cdot \frac{m_{1} m_{2}}{m_{1}+m_{3}} \cdot \theta,
$$

or $c^{\prime}$ is proportional to the square root of the temperature. We may therefore express the number of blows $>c$ in the form

$$
2 \mathrm{NN}^{\prime} s^{2}\left(\frac{m_{1}+m_{2}}{m_{1} m_{2}}\right) c^{\prime} e^{-\frac{c^{2}}{c^{\prime 2}}}
$$

\section{Case of an Elementary Gas.}

11. We will now employ the foregoing formula to investigate the effect of dissociation on an elementary gas whose molecule is diatomic. We shall suppose the dissociation to occur through a molecule receiving a blow $>c$; also that if two atoms come within a mean distance $s_{2}$ so that they would impinge with a blow $<c$, they will combine into a molecule.

Let then $x=$ number of molecules in unit of volume,

$2 y=$ number of free atoms in unit of volume, so that $2(x+y)=$ whole number of atoms present $=\mathbf{N}$, say.

Further, let $m=$ mass of an atom;

$s, s_{1}, s_{2}$ be the mean effective distances between two molecules, a molecule and an atom, and two atoms.

Then the number of collisions of molecules with a blow $>c$

$$
=2 x^{2} \sqrt{\pi} s^{2} \sqrt{\frac{\bar{\theta}}{m}} e^{-\frac{c^{2}}{4 m \theta}} .
$$

$=$ half the number of molecules destroyed The number of 
collisions of a molecule and atom with a blow $>c$

$$
=4 x y \sqrt{\pi} s_{1}^{2} \sqrt{\frac{3 \theta}{2 m}} e^{-\frac{3 c^{2}}{8 m \theta}}
$$

$=$ the number of molecules destroyed. The number of collisions of two atoms with a blow $<c$

$$
=8 y^{2} \sqrt{ } \pi s_{2}^{2} \sqrt{\frac{2 \theta}{m}}\left(1-e^{-\frac{c^{2}}{2 m \theta}}\right) .
$$

Hence the whole gain of molecules in a unit of time is $\frac{d x}{d t}=\left\{2 y^{2} s_{2}^{2} \sqrt{2}\left(1-e^{-\frac{c^{2}}{2 m \theta}}\right)-x y s_{1}^{2} \sqrt{\frac{3}{2}} e^{-\frac{3 c^{2}}{8 m \theta}}-x^{2} s^{2} e^{-\frac{c^{2}}{4 m \theta}}\right\} 4 \sqrt{\frac{\pi \theta}{m}}$ When the temperature is constant $\frac{d x}{d t}$ must $=0$, which condition, with $2(x+y)=\mathrm{N}$, gives us equations to find $x$. Putting

the condition becomes

$$
\frac{x}{y}=\xi, \quad \frac{c^{2}}{4 m}=\theta_{0}
$$

$$
\xi^{2}+\xi \cdot \sqrt{\frac{\overline{3}}{2}}\left(\frac{s_{1}}{s}\right)^{2} e^{-\frac{\theta_{0}}{2 \theta}}-2 \sqrt{2} \cdot\left(\frac{s_{2}}{s}\right)^{2}\left(e^{\frac{\theta_{0}}{\theta}}-e^{-\frac{\theta_{0}}{\theta}}\right)=0 ;
$$

say

whence

$$
\xi^{2}+2 a \xi-b=0
$$

$$
\xi= \pm \sqrt{a^{2}+b}-a
$$

12. The negative root has no meaning, whence we must take the positive root. But before we can assert that this value of $\xi$ gives the actual proportion, we must see whether the state of the gas would be stable. The condition for this is clearly that, if $x$ be increased, $\frac{d x}{d t}$ must be negative, and vice versâ. Writing

$$
\frac{d x}{d t}=f(x, y), \quad x+y=\frac{\mathrm{N}}{2},
$$

we have when $x$ is increased to $x+h$, and therefore $y$ to $y-h$,

$$
\frac{d x}{d t}=f(x y)+h\left(\frac{d f}{d x}-\frac{d f}{d y}\right)=h\left(\frac{d f}{d x}-\frac{d f}{d y}\right) ;
$$

we must therefore have $\frac{d f}{d x}-\frac{d f}{d y}=$ negative quantity. In this particular case

$$
f(x y)=\lambda\left(b y^{2}-2 a x y-x^{2}\right)
$$

where $\lambda, a, b$ are positive. The condition for stability, there- 
fore, is that $-x-a y-b y+a x$ must be negative, or

or, substituting for $\xi$

$$
\xi+\overline{a+b}>a \xi
$$

$$
1>a-\sqrt{a^{2}+b},
$$

which is clearly the case, and the value of $\xi$ therefore gives a stable state.

The condition may be stated in a different form as follows. When $x$ increases through the root, $f(x y)$ must change from positive to negative. From this again we see that the positive root of $b y^{2}-2 a x y-x^{2}=0$ gives a stable state. For when $x=0$ the expression is positive, and when $x=\infty$ it is negative, whence, as there is only one positive root, $f(x y)$ must change from positive to negative as $x$ increases through it.

13. The expression above found for $\xi$ gives twice the ratio of molecules to free atoms in the gas at any given temperature $\theta$. The proportion of molecules to the whole number of moving particles in the gas is therefore $=\frac{\xi}{2+\xi}=\eta$ suppose whilst the whole number of moving particles $=2 y+x=\frac{1}{2} \frac{2+\xi}{1+\xi} \mathrm{N}$. In order to obtain some idea of the law of variation of the proportions of molecules and free atoms with the temperature, I have traced the curve in fig. 1 . The abscisse denote the tem-

Fig. 1.

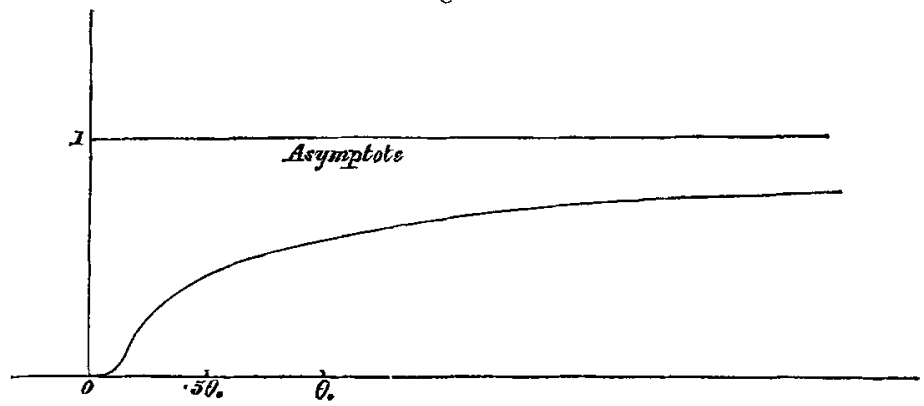

perature measured in terms of $\theta_{0}$, whilst the ordinates give the proportion of free atoms to moving particles. The particular curve represented belongs to the case where $s_{1}=\frac{s+s_{2}}{2}$ (which seems very likely the case) and $s=\frac{3}{2} s_{2}$ (or the radius of action of a molecule equal to the sum of the radii of action of the atoms of which it is composed); but the general form of the curve does not depend on the values of $s, s_{1}, s_{2}$, and only varies very slightly with their variations. It is noticeable that there 
are two periods when the variation of the proportions is small, viz. when the temperature is small compared with $\theta_{0}$ (less than $\frac{1}{10} \theta_{0}$ ) and when it is greater than $\theta_{0}$. The curve, of course, has an asymptote at a distance 1 from the origin; if we regard this as the line of abscissæ, the ordinates measured from it give the values of $\eta$.

14. In considering the pressure of the gas we may treat it as a mixture of two. We shall therefore have

$$
p=\frac{2}{3} \cdot 2 m x v_{1}^{2}+\frac{2}{3} m \cdot 2 y v_{2}^{2}
$$

where $v_{1}, v_{2}$ are the velocities of mean square in the two cases; and therefore $2 m v_{1}^{2}=m v_{2}^{2}=\lambda \theta$, where $\theta$ is the absolute temperature and $\lambda$ some constant, whence

$$
\begin{aligned}
p=\frac{2}{3}(x+2 y) \lambda \theta=\frac{\mathrm{N}}{3} \frac{(2+\xi)}{1+\xi} \lambda \theta=\frac{\mathrm{N}}{3}\left(1+\frac{1}{1+\xi}\right) \lambda \theta \\
=\kappa(1+\zeta) \rho \theta, \text { say. }
\end{aligned}
$$

If there were no dissociation, we should have, calling $\mathrm{P}$ the pressure in this case,

hence

$$
\mathrm{P}=\kappa \rho \theta ;
$$

$$
p=(1+\zeta) \mathrm{P} \text {. }
$$

Fig. 2 shows the relations of $p$ and $\mathrm{P}$ in a gas where $s=\frac{3}{2} s_{2}$;

Fig. 2.

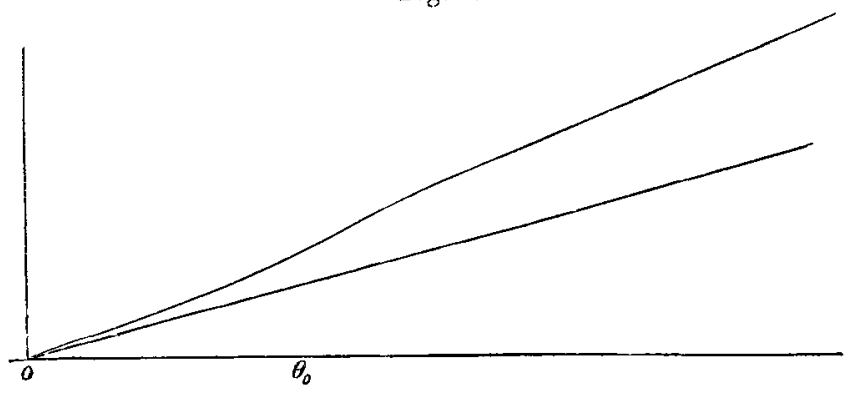

the abscissæ represent the temperature, and the ordinates the corresponding pressures in the two cases. The bend in the curve between $\cdot 5 \theta_{0}$ and $1.5 \theta_{0}$ is noticeable.

15. According to the ordinary theory $\mathrm{P}=\kappa \rho t$, where $t$ is the temperature measured from a certain zero-point, which is very slightly different for the different permanent gases. According to the above theory $p=\kappa \rho(1+\zeta) \theta$, where $\theta$ is the mean kinetic energy of translation of the particles composing the gas. Now, in the formula $\mathrm{P}=\kappa \rho t$, the temperatures are 
measured by comparison with the temperature of mercury or some other substance; and all we can deduce is that when the temperature increases a certain degree, the pressures of all the permanent gases increase in very nearly the same ratio with one another; but we have no proof that what we consider equal increments of temperature corresponds to exactly equal increments of mean kinetic energy. This is generally acknowledged and the temperatures measured by the "airthermometer" are supposed to be correct, whilst the variations from the law $\mathrm{P}=\kappa \rho t$ (when $t$ is measured by the mercurythermometer) are set down to the account of the unequal expansibility of mercury at different temperatures. But even on this supposition all that experiment tells us is that, in the formula $\mathrm{P}=\kappa \rho t, \kappa$ is the same for all permanent gases, and $t$ is measured from nearly the same zero-point. For instance, suppose that $\zeta$ is the same for all gases at the same temperature ; then, $(1+\zeta) \theta$ being the same at the same temperature for all gases, we should find in our experiments that $\kappa$ is the same and $t$ is measured from the same zero. But if we could by some means measure $\theta$ correctly and then apply our corrections to the temperatures as given by the mercurythermometer, we should find that the coefficient of expansion of mercury increases still more with the real temperature than is apparent from our comparisons of air and mercury, supposing, as is done, that equal increments of the pressure of air correspond to equal increments of temperature, and not, as on the theory of dissociation, to equal increments of the quantity $(1+\zeta) \theta$.

Since it is highly probable that the relations between $s, s_{1}, s_{2}$ are the same for all gases, the only condition that $(1+\zeta) \theta$ may be the same is that $\theta_{0}$ or $\frac{c^{2}}{m}$ may be the same, which would mean that, if two molecules are to break up, the relative velocity of the two impinging directly must be such-not that the minimum blow is the same in all gases, but that the vis viva must be the same, or that the force of cohesion is proportional to the square root of the mass. As I see no likely hypothesis on which this may be the case, I think it is not probable that $(1+\xi) \theta$ is rigorously independent of the kind of gas. But that the theory may agree with experiment, it is not necessary that $(1+\zeta) \theta$ should be rigorously the same for all gases; in fact, experiment tells us it is not so, although the variation is very small.

16. If for all the permanent gases $\theta_{0}$ be small compared with our ordinary temperatures, then our ordinary temperatures are some multiples of $\theta_{0}$, and the range within which 
experiments have been made is also some multiple of $\theta_{0}$. Moreover a slight difference in the values of $\theta_{0}$ for the different gases would give a greater difference in the law of pressure as the temperature increases. If, on the other hand, $\theta_{0}$ be great, so that ordinary temperatures are $<\frac{1}{10} \theta_{0}$, then as we have seen before, the variation and dissociation is small, and the more so as the maximum range of the Centigrade temperatures would all be small fractional parts of $\theta_{0^{\circ}}$. Here also variations of $\theta_{0}$ would not produce much difference between different gases at ordinary temperatures. We should be led therefore to suppose that $\theta_{0}$ is in general large. This is also supported by the fact that it is highly probable that at ordinary temperatures the dissociation of the permanent gases is small. For the sake of illustrating this and also to obtain some idea of the magnitude of the variations involved, we will consider more fully the case of two gases, in one of which $\theta_{0}=100^{\circ}$, and in the other $=5000^{\circ}$, the degrees being Centigrade and measured from absolute zero, i. e. $-273^{\circ} \mathrm{C}$.

17. In Tables I. and II. below, column 1 gives the temperature; column 2 the dissociation at the given temperature, the dissociation being measured by the proportion of free atoms to moving particles in the gas; column 3 gives the value of $1+\zeta$ in the formula $p=\kappa \rho(1+\xi) \theta$, and which we may call the expansibility; whilst column 4 gives the ratio $\frac{\frac{p}{\theta}}{\frac{p_{0}}{\theta_{0}}}\left(p_{0}, \theta_{0}\right.$ being the pressure and temperature at $27^{\circ} \mathrm{C}$. $)$.

'TABLe I. $\left(\theta_{0}=-173^{\circ} \mathrm{C}.\right)$.

\begin{tabular}{|c|c|c|c|}
\hline 0. & $\frac{2 y}{x+2 y}$ & $1+\zeta$. & $\frac{\frac{p}{\theta}}{p_{0}}$ \\
& & & $\frac{\theta_{0}}{\theta^{2}}$ \\
\hline & & & \\
-273 & 0 & 1 & \\
-173 & .610 & 1.439 & 0.873 \\
-73 & .728 & 1.571 & 0.953 \\
27 & .785 & 1.6471 & 1 \\
127 & .820 & 1.695 & $1 \cdot 029$ \\
527 & .845 & 1.732 & 1.051 \\
$\infty$ & 1 & 1.752 & 1.063 \\
\hline
\end{tabular}


TABLe II. $\left(\theta_{0}=5000^{\circ}=4727^{\circ}\right.$ C. $)$.

\begin{tabular}{|c|c|c|c|}
\hline$\theta$. & $\frac{2 y}{x+2 y}$ & $1+\zeta$. & $\frac{\frac{p}{\theta}}{\frac{p}{p_{0}}}$ \\
\hline-273 & 0 & 1 & \\
\hline-73 & .0000 & $1 \cdot 0000$ & \\
\hline 27 & .0017 & 1.0008 & 1 \\
\hline 127 & .0096 & $1 \cdot 0048$ & $1 \cdot 0048$ \\
\hline 227 & $\cdot 0269$ & 1.0136 & $1 \cdot 0136$ \\
\hline 327 & .057 & 1.0268 & 1.0260 \\
\hline 527 & $\cdot 116$ & $1 \cdot 0617$ & 1.0610 \\
\hline 727 & 182 & $1 \cdot 1003$ & $\overline{1} \cdot 0994$ \\
\hline 1227 & $\cdot 313$ & $1 \cdot 1850$ & $1 \cdot 1841$ \\
\hline 1227 & $\begin{array}{l}459 \\
.459\end{array}$ & 1.2970 & $1 \cdot 2961$ \\
\hline$\infty$ & 1 & 2 & 1.9984 \\
\hline
\end{tabular}

18. From Table II. we see that at ordinary temperatures the dissociation is very small when $\theta_{0}=5000^{\circ}$, even up as high as $700^{\circ} \mathrm{C}$. ; whilst when $\theta_{0}=100^{\circ}$ or $-173^{\circ} \mathrm{C}$., the dissociation is always large, even down to $-200^{\circ} \mathrm{C}$. Also the expansibility in the first case between temperatures $0^{\circ} \mathrm{C}$. and $100^{\circ} \mathrm{C}$. varies as much as in the second case between $0^{\circ} \mathrm{C}$. and $300^{\circ} \mathrm{C}$, though when we get to high temperatures the expansibility changes more slowly in the first case than in the second. In the second case the expansibility is almost constant between $0^{\circ} \mathrm{C}$. and $100^{\circ} \mathrm{C}$., and even up to $300^{\circ}$ changes only slightly. Again, a difference of 10 per cent. in the value of $\theta_{0}$ in different gases will produce a difference in their expansibilities at ordinary temperatures of $\cdot 004$ when $\theta_{0}$ is small and about $-173^{\circ} \mathrm{C}$., and of $\cdot 00065$ when $\theta_{0}$ is large and about $4727^{\circ} \mathrm{C}$. In other words, if $\theta_{0}$ be $-173^{\circ} \mathrm{C}$. in one gas and the value of $\theta_{0}$ in the other gas vary to the extent of $10^{\circ}$, the difference in their expansibilities will vary to the extent of .004 ; whilst if in one $\theta_{0}$ be $4727^{\circ} \mathrm{C}$. and the value of $\theta_{0}$ in the other vary to the extent of $500^{\circ}$, the difference in their expansibilities will vary to the extent of $\cdot 00065$. These three reasons, viz. (1) smallness of dissociation, (2) constancy of expansibility, (3) near equality of expansibilities of different gases, lead us to believe that in the case of the permanent gases $\mathscr{\theta}_{0}$ is large compared with ordinary temperatures. Still there may be gases whose $\theta_{0}$ is small. It is possible that mercury vapour is such a gas, and that this accounts for the fact that its molecules are monatomic, viz. that the temperature at which it exists is a large multiple of its $\theta_{0}$, and that therefore the dissociation is large. 
19. In considering the effect of dissociation on the specific heats of gases, we may not treat them as a mixture of two whose proportions remain constant; for in raising the temperature, not only is work done in increasing the kinetic energy of translation and internal motion, but also in destroying a certain proportion of molecules. Let, as before, $x$ be the number of molecules, $2 y$ the number of atoms present when the temperature is $\theta$. Let $\lambda_{1}, \lambda_{2}$ be the ratios of the whole energy to that of translation in molecules and free atoms respectively. Then we have, if $Q$ is the quantity of energy present in unit of volume, and I the potential energy of combination of a single molecule,

$$
\begin{aligned}
\dot{Q} & =\frac{1}{2} 2 m v_{1}^{2} \cdot \lambda_{1} x+\frac{1}{2} m r_{2}^{2} \cdot 2 \lambda_{2} y+\mathrm{I} x \\
& =\left(\lambda_{1} x+2 \lambda_{2} y\right) \lambda \theta+\mathrm{I} x .
\end{aligned}
$$

Let a quantity $\delta \mathrm{Q}$ of heat be applied, the rolume being constant. Then

$$
\begin{aligned}
\delta Q= & \left\{\left(\lambda_{1} \frac{d x}{d \theta}+2 \lambda_{2} \frac{d y}{d \theta}\right) \lambda \theta+\left(\lambda_{1} x+2 \lambda_{2} y\right) \lambda+1 \frac{d \cdot x}{d \theta}\right\} d \theta \\
& =c d \theta,
\end{aligned}
$$

where $c$ is the specific heat at constant volume. Also

$$
\begin{aligned}
& 2(x+y)=\mathrm{N} ; \\
& \therefore \frac{d x}{d \theta}+\frac{d y}{d \theta}=0 .
\end{aligned}
$$

And

$$
c=\left\{\left(-\lambda_{1}+2 \lambda_{2}\right) \theta \frac{d y}{d \theta}+\lambda_{1} x+2 \lambda_{2} y-\frac{\mathrm{I}}{\lambda} \frac{d y}{d \theta}\right\} \lambda ;
$$

and if $c^{\prime}$ is the specific heat at constant pressure,

$$
c^{\prime}=c+p \frac{d v}{d \theta} \text {. }
$$

Now $p=\frac{\mathrm{N}}{3}(1+\zeta) \lambda \theta$.

If $\mathrm{V}$ be the volume occupied by the gas and $\mathrm{N}^{\prime}$ the number of atoms in it,

$$
\mathrm{N}=\frac{\mathrm{N}^{\prime}}{\mathrm{V}}
$$

Hence

$$
\begin{gathered}
p V=\lambda \frac{N^{\prime}}{3} \mid(1+\zeta) \theta ; \\
\therefore \quad p \frac{d V}{d \theta}=\lambda \frac{N^{\prime}}{3}\left\{1+\zeta+\theta \frac{d \zeta}{d \theta}\right\},
\end{gathered}
$$


416 Mr. W. M. Hicks on some Effects of Dissociation

where $p$ is constant;

$$
\begin{aligned}
& \therefore c^{\prime}=c+\frac{\lambda \mathrm{N}}{3}\left(1+\zeta+\theta \frac{d \zeta}{d \theta}\right), \\
& \frac{c^{\prime}}{c}=1+\frac{\mathrm{N}}{3} \frac{\theta \frac{d \zeta}{d \theta}+1+\zeta}{\left(2 \lambda_{2}-\lambda_{1}\right) \theta \frac{d y}{d \theta}+\lambda_{1} x+2 \lambda_{2} y-\frac{1}{\lambda} \frac{d y}{d \theta}} \\
& =1+\frac{2}{3} \frac{\theta \frac{d \zeta}{d \theta}+1+\zeta}{\left(2 \lambda_{2}-\lambda_{1}\right) \theta \frac{d \zeta}{d \theta}+\lambda_{1}+\left(2 \lambda_{2}-\lambda_{1}\right) \zeta-\frac{1}{\lambda} \frac{d \zeta}{d \theta}} .
\end{aligned}
$$

In the above we have neglected the variation of the potential energy of molecules except in the case where they are broken up. The above formula is greatly simplified if we consider, as is highly probable, that a molecule has twice the number of degrees of freedom of a free atom-in other words,

$2 \lambda_{2}=\lambda_{1}$; we then get

$$
\frac{e^{\prime}}{i}=1+\frac{2}{3} \frac{1+\zeta+\theta \frac{d \zeta}{d \theta}}{\lambda_{1}-\frac{1}{\lambda} \frac{d \zeta}{d \theta}}
$$

20. If the atoms be smooth and spherical (as has been supposed throughout the present paper), any internal energy an atom may have must be unaffected by change of temperature; and in this case $\lambda_{1}=2$.

The blow just sufficient to break up a molecule is $c$; if this acted in the most favourable manner, the work done in separating a molecule would be $\frac{c^{2}}{2 m}=2 \lambda \theta_{0}$. Hence $\mathrm{I}$ is $<2 \lambda \theta_{0}$. If we put $I=2 \lambda \theta_{0}$,

$$
\begin{aligned}
\frac{c^{\prime}}{c} & =1+\frac{2}{3} \frac{1+\zeta+\theta \frac{d}{d \theta}}{2-2 \theta_{0}} \\
& =1+\frac{1}{3} \frac{1+\zeta+\theta \frac{d}{d \theta}}{1-\theta_{0} \frac{d}{d \theta}} .
\end{aligned}
$$

If $\theta_{0}$ is small and about $100^{\circ}, i_{.}$e. $-173^{\circ} \mathrm{C}$, we find from 
on the Physical Properties of Gases.

Table I. that, at a temperature of about $70^{\circ} \mathrm{C}$,

$$
1+\zeta=1 \cdot 671, \quad \frac{d \zeta}{d \theta}=\cdot 00048, \quad \theta=350 ;
$$

whence it follows that

$$
\frac{e^{\prime}}{c}=1 \cdot 609 \text {. }
$$

If $\theta_{0}$ is large and about $5000^{\circ}$ or $4727^{\circ} \mathrm{C}$., Table II. gives us for a temperature of $70^{\circ}$

whence

$$
1+\zeta=1 \cdot 0028, \frac{d \zeta}{d \theta}=\cdot 00004,
$$

$$
\frac{c^{\prime}}{c}=1 \cdot 423
$$

In the above we have confessedly taken I too large, as we showed that $2 \lambda \theta_{0}$ was its superior limit; if we were to take $\mathrm{I}=2 \lambda \theta_{0} \times \cdot 4$, we should get

$$
\frac{c^{\prime}}{c}=1 \cdot 403 \text {. }
$$

The value of $\frac{c^{\prime}}{c}$ for the permanent gases is about $1 \cdot 408$.

Not only does this result confirm our former conclusion that $\theta_{0}$ is very large, but it adds largely to the probal,ility of the general theory here set forth, since the great difficulty of the kinetic theory of gases hitherto has been to explain the value of $\frac{c^{\prime}}{c}$ for the permanent gases whose molecules are diatomic. Mercury vapour which has simple molecules, or is monatomic, and which therefore has no dissociation, has the value given by the ordinary theory, as has been shown recently by Messrs. Kundt and Warburg.

21. To satisfy experience, it is further necessary to show that $c$ does not vary much within the ordinary range of temperature. We have

$$
\begin{aligned}
c & =\frac{\lambda N}{2}\left\{\left(2 \lambda_{2}-\lambda_{1}\right)\left(\theta \frac{d \zeta}{d \theta}+\zeta\right)+\lambda_{1}-\frac{I}{\lambda} \frac{d \zeta}{d \theta}\right\} \\
& =\frac{\lambda N}{2}\left\{\lambda_{1}-2 \kappa \theta_{0} \frac{d \zeta}{d \theta}\right\},
\end{aligned}
$$

where $\boldsymbol{\kappa}$ is a fraction. Hence

$$
\frac{d c}{d \theta}=-\lambda \kappa \mathrm{N} \theta_{0} \frac{d^{2} \zeta}{d \theta^{2}} \text {. }
$$

Phil. Mag. S. 5. Vol. 3. No. 20. June 1877. $2 \mathrm{E}$ 
418 Mr. R. H. M. Bosanquet on the Theory of Sound.

Now $\frac{d \zeta}{d \theta}$ is throughout very small, and varies very slowly, so that $\frac{d^{2} \zeta}{d \theta^{2}}$ is extremely small. In fact, from Table II. we see that at $100^{\circ} \mathrm{C} \cdot \frac{d^{2} \zeta}{d \theta^{2}}$ is somewhere about $\cdot 00000048$.

[To be continued.]

LVI. Notes on the Theory of Sound. By R. H. M. Bosanquet, Fellow of.St. John's College, Oxford.

[Continued from p. 349.]

4. On Combined Wave-systems.

THE principle that a stream of sound may be regarded as a flow of energy, which cannot of itself increase or diminish in quantity, enables us to deal with certain simple cases of combined wave-systems. I restrict myself for the present to the case of plane waves.

Prop. I.-If two equal and similar pendulum wave-systems, travelling in opposite directions, meet in air, they form a stationary wave which will carry the whole energy of both.

Let

$$
\begin{aligned}
& y_{1}=a \sin \frac{2 \pi}{\lambda}(v t-x), \\
& y_{3}=a \sin \frac{2 \pi}{\lambda}(v t+x)
\end{aligned}
$$

be the two equal systems travelling opposite ways; the more general equations can always be reduced to this form by suitable choice of the origins of space and time.

Then

$$
\begin{aligned}
\mathrm{Y} & =y_{1}+y_{2}=2 a \sin \frac{2 \pi v t}{\lambda} \cos \frac{2 \pi}{\lambda} x, \\
\frac{d \mathrm{Y}}{d t} & =\frac{4 a \pi v}{\lambda} \cos \frac{2 \pi v t}{\lambda} \cos \frac{2 \pi}{\lambda} x, \\
\frac{d \mathrm{Y}}{d x} & =-\frac{4 \pi a}{\lambda} \sin \frac{2 \pi v t}{\lambda} \sin \frac{2 \pi}{\lambda} x .
\end{aligned}
$$

When $t=0, \mathrm{Y}=0$ and $\frac{d \mathrm{Y}}{d x}=0$, or there is no displacement and no pressure anywhere along the stationary wave, but there is a maximum velocity,

$$
\frac{d \mathrm{Y}}{d t}=\frac{4 a \pi v}{\lambda} \cos \frac{2 \pi x}{\lambda}
$$

